\title{
Feasibility and effect of in-home physical exercise training delivered via telehealth before bariatric surgery
}

\author{
Aurélie Baillot ${ }^{1}$, Patrick Boissy ${ }^{2}$, Michel Tousignant ${ }^{2}$, Marie-France Langlois ${ }^{3}$ \\ ${ }^{1}$ Nursing Department, Université du Québec en Outaouais, Institut de recherche de \\ l'hôpital Montfort, Canada. \\ ${ }^{2}$ Research Centre on Aging, Health and Social Services Centre, Institute of Geriatrics, \\ Université de Sherbrooke, Canada. \\ ${ }^{3}$ Department of Medicine, Division of Endocrinology, Université de Sherbrooke, Research \\ Center of the Centre hospitalier universitaire de Sherbrooke, Canada.
}

Corresponding author: Aurélie Baillot, Nursing Department, Université du Québec en Outaouais, 283 boulevard Alexandre-Taché, C.P. 1250, Succursale Hull, Gatineau, (Québec) Canada J8X 3X7

Office: 00 1-819-595-3900 poste 1995; Fax: 00 1-819 595-2202 aurelie.baillot@uqo.ca

Running head: Telehealth exercise training and severe obesity Article type: Preliminary Communication 
Main text Words count: 2997

Abstract words count: 197 


\begin{abstract}
Optimal physical activity (PA) interventions are needed to increase PA in individuals with severe obesity, and optimize the results of bariatric surgery (BS). The aim of this study was to assess the feasibility and effect of in-home Pre-Surgical Exercise Training delivered via telehealth (TelePreSET) in subjects awaiting BS. Six women following the TelePreSET were compared to the women from a previous study (12 performing the PreSET in a gymnasium and 11 receiving usual care). In-home TelePreSET (12-weeks of endurance and strength training) was supervised twice weekly using videoconferencing. Physical fitness, quality of life, exercise beliefs, anthropometric measures, and telehealth perception were assessed before and after 12-weeks. Satisfaction was evaluated with questionnaires at the end of the intervention. The TelePreSET participants attended $96 \%$ of the exercise sessions, and were very satisfied by the TelePreSET. The baseline telehealth perception score was high, and increased significantly after the intervention. The TelePreSET group significantly increased their physical fitness compared to the usual care group. No significant change was noted in other outcomes. The TelePreSET is feasible and seems effective to improve the physical fitness of women awaiting BS. Further studies will confirm beneficial effects of this innovative mode of delivery.
\end{abstract}




\section{Keywords:}

Physical activity, severe obesity, physical fitness, telecare 


\section{Introduction}

Bariatric surgery is the most effective long-term treatment for patients with severe obesity with positive impacts on comorbid conditions and mortality (1). Regular physical activity (PA) is recommended by experts in the surgical management of individuals with severe obesity (2). Indeed, increasing evidence shows that PA interventions are valuable options for health improvements in this population before and after surgery $(3,4)$.

Most individuals with severe obesity have reduced physical fitness, associated with discomfort during exercise (5-8), which may limit their motivation and ability to engage in regular PA. Economic and psychosocial barriers may also be added to physical barriers, accentuating the lack of motivation $(4,9,10)$. Optimal PA interventions are needed to increase PA adoption in individuals with severe obesity and optimize the results of bariatric surgery (BS).

Pre-Surgical Exercise Training (PreSET) and PA counselling are feasible and effective to improve the PA level, quality of life, and physical fitness in individuals awaiting BS (3, 1114). Adding a PreSET to individual lifestyle counselling can also improve social interactions and decrease embarrassment during PA (14). However, the number of individuals awaiting BS that could be enrolled in such programs is often constrained as a 
lot of them are unable to attend regular supervised exercise sessions because of time and transportation constraints.

In-home telecare could be an interesting option to overcome these barriers, since it can be provided at home in real time using telecommunication networks. The rationale for providing in-home telecare is grounded in minimizing the barriers of distance, time, and cost, and increasing accessibility to health services for people who are disadvantaged because of economical or functional limitations (15). For example, results have shown that in-home telecare in rehabilitation can improve the quality of health care and be as effective as face to face meeting to improve the health of patients (16-25). The attendance rates, treatment adherence and patient satisfaction for telerehabilitation are very high, although few comparisons are available with other types of interventions $(17,19)$. While telerehabilitation has been used in different patient populations (17, 22, 26-29), to our knowledge no study has focused in this mode of delivery to supervise exercise training in individuals with severe obesity. Thus, the aim of this study was to assess the feasibility and effect of a 12-week in-home Pre-Surgical Exercise Training delivered via telehealth (TelePreSET) in subjects awaiting BS. 


\section{Materials and Methods}

\section{Design and subjects}

A pre/post-test design with historical comparison groups was used to assess the feasibility and effect of a 12-week in-home Pre-Surgical Exercise Training delivered via telehealth. The study protocol was approved by the Institutional Ethics Review Board of research on humans of the CHUS and Université de Sherbrooke (project 13-209) and each subject provided written informed consent to participate in the study. This trial was registered at clinicaltrials.gov (NCT02083913). Six women awaiting BS (aged $\geq 18$ years; body mass index (BMI) $\geq 35$ with comorbidities or $\geq 40 \mathrm{~kg} / \mathrm{m}^{2}$ ), who were expected to be operated within 3 to 6 months in our institution, and had sufficient space at home for exercise training $\left(4 \mathrm{~m}^{2}\right)$ were included in the study. Exclusion criteria were: medical contraindication for PA, functional limitations (unable to walk at least 5 minutes), > one weekly supervised exercise sessions, no access with any vendor to high speed residential Internet, and not understanding French. Five women for BS refused to be contacted, and 21 were contacted by telephone between March and October 2014. Among the 21 women, 12 refused to participate (no time for exercise training $n=7$; moving $n=2$; not interested by research or telehealth $n=3)$, and 9 were excluded due to insufficient space for training $(n=5)$, functional limitation $(n=1)$, current involvement in supervised exercise $(n=2)$, and inability to 
understand French $(\mathrm{n}=1)$. The TelePreSET participants were compared to the female participants from a previous study: 12 women performing the PreSET in a gymnasium and 11 women, who received only lifestyle intervention (usual care group)(14).

\section{Interventions and teleheath system}

In addition to usual care (individual lifestyle counselling sessions every 6-8 weeks), participants underwent two weekly 80-minutes sessions of endurance and strength training supervised in telehealth and one weekly unsupervised session for 12 weeks. The detailed exercise modalities have been descried in our previous study offering supervised exercise session in gymnasium and swimming pool (11). We could not offer aquagym, and the strength training loads progressed from 2 to $18 \mathrm{lbs}$ according to the muscular group worked, which is higher than our former study (11). Arm-ergocycle, steps, dumbbells, elastic bands, medicine ball $(2 \mathrm{~kg})$ and electronic pulse monitor (polar $\left.{ }^{\circledR}\right)$ were lent to the participants. Treadmill, cyclo-ergometer and elliptical owned by the participants were used when they were available. Supervised sessions were provided one to one using a videoconferencing based telecare platform developed and tested previously $(20,23,24)$. The platform on the kinesiologist and patient sides uses an all in one computer (HP Touch Smart) running an h.264 software coder-decoder (CODEC) connected to a pan-tilt-zoom 
(PTZ) camera with an omnidirectional microphone. On the kinesiologist side, a dedicated software (TeRa) is added to the videoconference link to enable user-friendly control and monitoring of sessions, near and far end camera controls (pan, tilt, zoom), picture in picture and reverse picture in picture display of camera sources. Depending on the presence or absence of an internet connection at the home of the participants and on the bandwidth available, arrangements were made to schedule installation/activation or upgrade of services for the duration of the project. After installation or upgrade of the Internet service, a technician from the research team visited the patient's home to 1) analyze the placement of the equipment; 2) create or expand a secured wireless network connecting the videoconferencing system to the existing Internet connection, 3) install and configure the different components of the system; 4) test connection between the remote clinical site system and the patient's home system; 5) assess the bandwith and reliability of the internet connection and 6) train the patient on the sequence of operation of the system. The platform was installed before the start of the TelePreSET in the subject's home and removed at the end of the intervention. 


\section{Sociodemographic, anthropometric and medical variables}

Height, neck circumference, body and fat mass were measured using standard protocol (11). Blood pressure and HR were measured after 5 minutes of rest in sitting position with an automatic blood pressure device (Omron HEM 741 ${ }^{\circledR}$ ) (14). Comorbidities were extracted from medical charts. The baseline PA level (activity at work, travel, and recreational activities) was evaluated with the Global PA Questionnaire, developed by World Health Organisation and comprising 16 questions (30). A total PA MET-min/week score was calculated and participants were classified as following PA recommendations

(600 MET-min/week) according to the WHO guideline (http://www.who.int/ chp/steps/resources/GPAQ_Analysis_Guide.pdf).

\section{Compliance}

The supervised exercise session attendance was calculated by dividing the number of sessions realized with supervision by the total number of supervised sessions proposed until the final assessment (2x/week). The total exercise sessions attendance was calculated by dividing the number of exercise sessions realized (with or without supervision) by the number of sessions required (3/week) until the final assessment. (11). 


\section{Satisfaction}

The validated in-home telehealth patients' perception questionnaire was used to evaluate the participants' telehealth perception (Cronbach's $\alpha$ coefficient $=0.80)(31,32)$. Seventeen questions on a 5-point Likert scale give a total score, converted in percentage. The closer the score is to 100 , the better is the telehealth perception.

The TelePreSET' satisfaction was evaluated at the end of the intervention with a validated questionnaire (Cronbach's $\alpha$ coefficient $=0.93)(33)$. It contains 14 questions on a 5-point Likert scale. A total score, converted in percentage was used to reflect global satisfaction, and three subscores were computed: quality of care provided, similarity to face to face encounter, and perception of the interaction.

The healthcare satisfaction questionnaire was used to evaluate the participants' satisfaction with healthcare services received during the TelePreSET (34). It has a good internal consistency (Cronbach's $\alpha$ coefficient $=0.92)(34)$. Twenty-three questions are divided into 3 factors: satisfaction with the relationship with the healthcare professional, satisfaction with the services delivered, and satisfaction with the general healthcare organization. These three factors were also computed in a total score in percentage with the higher score representing the higher level of satisfaction. 


\section{Physical fitness}

Maximal aerobic capacity was assessed with a treadmill symptom-limited cardiac exercise test (Cornell $0,5,10)(35)$. The 6-minute walk test $(6 \mathrm{MWT})$ was used to assess functional capacity (36). Heart rate was assessed throughout the 6MWT with an Electronic pulse monitors (Polar $\mathrm{F}^{\mathrm{TM}}$ ). Heart cost, that reflects relative exercise intensity, was calculated using the 6MWT distance divided by 6MWT mean HR, as previously reported (11). The Sit to stand test, the Half-squat test and the Arm curl test were performed to evaluate muscular endurance of the upper and lower limbs (11).

\section{Weight-related quality of life (WRQOL) and physical exercise beliefs}

The Laval questionnaire and the physical exercise belief questionnaire were used to assessed WRQOL, and PA benefits and psychological barriers $(11,37,38)$.

\section{Statistical analysis}

Given the small sample size, non-parametric analyses were conducted. Baseline characteristics of the three groups were compared with Kruskal-Wallis test for continous data or Chi-square tests for nominal data (or Fisher's exact for data with less than 5 in any cell). The time effect of training was tested with the Wilcoxon tests in each group. The 
TelePreSET 12-weeks changes were compared with those of the two other groups with Mann-Whitney tests. The median values $\left(25-75^{\text {th }}\right.$ percentile) were used to present results. Data were analyzed with SPSS version 22.0 (SPSS Inc., Chicago, IL). The null hypothesis was rejected at $\mathrm{p}<0.05$.

\section{Results}

\section{Baseline characteristics and intervention compliance}

Baseline characteristics of the three groups are presented table 1. No significant difference was observed between groups. No drop out, injury or accident in the TelePreSET was noted. The TelePreSET participants attended more supervised exercise sessions compared to the PreSET participants [95.8 (85.1-100) vs. 80.1 (42.6-90.1) \%; $\mathrm{p}=0.02]$. No significant difference was found between these two groups concerning the attendance rate of the total exercise sessions [88.9 (68.7-93.8) vs. $62.4(38.2-87.8)) \%$; $\mathrm{p}=0.12]$.

\section{Satisfaction}

The baseline in-home telehealth patients' perception score was high [83.5 (80.9-91.2], and increased significantly after the intervention [90.0 (86.8-94.1); $\mathrm{p}=0.03]$. The global TelePreSET' satisfaction was 93.4 (89.3-97.7) \%, with subscores of 97.5 (91.9-98.1) \% for 
quality of care provided, $90.0(78.0-97.0) \%$ for similarity to face to face encounter, and 100.0 (95.0-100.0) \% for perception of the interaction. Participants always answered that they "totally agree" or "agree", except for these 3 questions: I can easily talk to my healthcare provider (disagree $n=1$ ); I can hear my health-care provider clearly (disagree $n=2$ ); I do not need assistance while using the system (disagree $n=1$; totally disagree $n=1$ ). The total participants' satisfaction with healthcare services received during the TelePreSET was 85.6 (76.3-95.8) \%, with subscores of $92.4(84.7-97.4) \%$ for the relationship with the healthcare professional, $82.7(67.2-97.4) \%$ for satisfaction with the services delivered and $85.6(76.3-95.8) \%$ for satisfaction with the general healthcare organization.

\section{2-week absolutechanges}

Table 2 presents baseline and 12-week physical fitness absolute changes in each group. Analyses showed no significant difference in changes of WRQOL $(p=0.9)$ and physical exercise beliefs $(\mathrm{p} \geq 0.2)$ between the TelePreSET and the PreSET groups. No difference was also found between the TelePreSET and the usual care groups ( $\mathrm{p} \geq 0.4)$, with a trend for difference in the confidence during exercise $[7.5(2.5-10.0)$ vs. $0.0(-15.0-5.0) ; p=0.06]$ and social interaction scores $[11.2(1.0-28.6)$ vs. $2.0(-10.2-2.0) ; \mathrm{p}=0.05]$. No significant change 
was found in anthropometric, body composition and blood pressure after 12 weeks in each group, and no significant difference between groups were observed ( $\mathrm{p}>0.2)$.

\section{Discussion}

The attendance of the TelePreSET group was excellent and greater than the PreSET group. Indeed, exercise sessions in the TelePreSET group were performed in-home and according to participants' schedule and could be re-scheduled when possible, while PreSET sessions were performed in a gymnasium in the evening with a strict schedule. Satisfaction is an important outcome to consider since it influences treatment compliance and motivation (39). For the TelePreSET intervention satisfaction level was high regarding each factor, in accordance with other studies offering telerehabilitation $(17,19)$. We noticed that the audio quality could be improved, but it didn't seem to have an impact of the use and acceptance of the participants, since the baseline level of telehealth perception was high, and the intervention increased it.

In accordance with our previous studies with in hospital-group exercise sessions $(11,14)$, we found that 12-weeks of TelePreSET significantly improved the 6MWT distance and the 
number of arm curl and sit to stand repetitions compared to the usual care group. However, no significant WRQOL and physical exercise beliefs changes was noted after the TelePreSET, in contrast with previous results $(11,14)$. This absence of effect may be explained by the lack of statistical power and/or patient interactions during sessions.

\section{Practical considerations}

To our knowledge, this is the first study that provided exercise training with a large variety of strength exercises (dumbells, medicine ball, elastic bands) and endurance activities (step, aerobic, treadmill, ergocycle) in the context of in-home telehealth. The challenge was to adapt the PreSET performed face-to-face in a gymnasium to a mode of communication over videoconferencing in different home environments. In the context of audio video mediated communication during in-home telecare, there are marked changes in the technical and interpersonal contexts within which communication takes place that can affect usability of providing in-home telecare. PA specialists had thus to be trained before the start of the TelePreSET to adapt exercises and interactions to these environments and context of communication: reduced space to exercise, no contact to correct movements, reduced visual range, desynchronization between sounds and video for fast movements, speaking alternately, and less visual details to observe participants exertion. 


\section{Clinical application}

In-home supervised exercise training via telehealth could provide a better access to exercise training for patients compared to in-hospital sessions. However, to provide optimal intervention, certain requirements in the participants' homes were pre-requisites (access to a high speed internet connection, adequate space for exercise, adequate lighting and temperature, quiet environment). Furthermore, TelePreSET intervention was not feasible with all patients (e.g: $24 \%$ of patients contacted had insufficient space for training). In addition, this option didn't remove totally time barriers to practice supervised exercise training according to the number of patients having refused to participate in the study for this reason $(\mathrm{n}=7 ; 33 \%)$.

In the context of supervised exercise training, telehealth group intervention has to be tested to reduce the cost of the intervention and lifestyle counselling could also be added to the intervention. Moreover, before implementing this kind of intervention in clinical practice, given the high non-participation rate (71\%), additional data are required in larger groups to assess the interest of patients awaiting BS. 


\section{Limitations}

For the interpretation of results some limitations have to be considered. First, the generalization of our results to all subjects awaiting BS is reduced due to the small sample size and the specificity of our sample. Indeed, our participants were women, having already received lifestyle intervention explaining probably the high rate of active subjects and motivated to practice PA. Then, no assessment of professional satisfactionwas performed. Finally, the TelePreSET satisfaction and perception rate results could have been skewed by

a desirability bias as they were obtained only for participants who accepted the intervention.

To conclude, our results suggested that a TelePreSET in women awaiting BS is feasible and seems effective to improve physical fitness. Further larger studies with randomized design and complete lifestyle intervention in telehealth are now required to confirm the benefits of this promising mode of delivery. 


\section{Acknowledgements}

Investigators would like to thank Dre Anne Méziat-Burdin, Dre Emilie Comeau and Amélie Laroche-Provencher for their collaboration in recruiting participants. We appreciate the contribution of Alexandrine Boucher, Sherbrooke University (Canada) kinesiology bachelor student for her help with the exercise training and data collection. In addition, we thank the engineer Simon Brière and the technician Antoine Guillerand for their technical help with the telehealth system. $A B$ is the recipient of a scholarship from the Department of Medicine of Université de Sherbrooke. PB and MFL are recipients of salary awards from the Fonds de recherche du Québec-Santé (FRQ-S).

Declaration of Conflicting Interests: No conflict

Funding: This study was supported by the Canadian Institutes of Health Research (CIHR, Grant No.OPB-131592). 


\section{References}

1. Sjostrom L. Review of the key results from the Swedish Obese Subjects (SOS) trial

- a prospective controlled intervention study of bariatric surgery. J Intern Med. 2013 Mar;273(3):219-34.

2. Mechanick JI, Youdim A, Jones DB, Garvey WT, Hurley DL, McMahon MM, et al. Clinical practice guidelines for the perioperative nutritional, metabolic, and nonsurgical support of the bariatric surgery patient--2013 update: cosponsored by American Association of Clinical Endocrinologists, The Obesity Society, and American Society for Metabolic \& Bariatric Surgery. Obesity. 2013 Mar;21:S1-27.

3. Baillot A, Audet M, Baillargeon JP, Dionne IJ, Valiquette L, Rosa-Fortin MM, et al. Impact of physical activity and fitness in class II and III obese individuals: a systematic review. Obes Rev. 2014 Apr 9;15(9):721-39.

4. King WC, Bond DS. The importance of pre and postoperative physical activity counseling in bariatric surgery. Exerc Sport Sci Rev. 2013;41(1):26-35.

5. Hulens M, Vansant G, Claessens AL, Lysens R, Muls E. Predictors of 6-minute walk test results in lean, obese and morbidly obese women. Scand J Med Sci Sports. 2003 Apr;13(2):98-105. 
6. Faintuch J, Souza SA, Valezi AC, Sant'Anna AF, Gama-Rodrigues JJ. Pulmonary function and aerobic capacity in asymptomatic bariatric candidates with very severe morbid obesity. Rev Hosp Clin Fac Med Sao Paulo. 2004 Aug;59(4):181-6.

7. Seres L, Lopez-Ayerbe J, Coll R, Rodriguez O, Manresa JM, Marrugat J, et al. Cardiopulmonary function and exercise capacity in patients with morbid obesity. Rev Esp Cardiol. 2003 Jun;56(6):594-600.

8. Gallagher MJ, Franklin BA, Ehrman JK, Keteyian SJ, Brawner CA, deJong AT, et al. Comparative impact of morbid obesity vs heart failure on cardiorespiratory fitness. Chest. 2005 Jun;127(6):2197-203.

9. Wiklund M, Olsén MF, Willén C. Physical activity as viewed by adults with severe obesity, awaiting gastric bypass surgery. Physiother Res Int. 2011;16(3):179-86.

10. Biddle SJ, Fox KR. Motivation for physical activity and weight management. Int J Obes Relat Metab Disord. 1998 Aug;22 Suppl 2:S39-47.

11. Baillot A, Mampuya WM, Comeau E, Meziat-Burdin A, Langlois MF. Feasibility and impacts of supervised exercise training in subjects with obesity awaiting bariatric surgery: a pilot study. Obes Surg. 2013 Jul;23(7):882-91.

12. Bond DS, Vithiananthan S, Graham Thomas J, Trautvetter J, Unick JL, Jakicic JM, et al. Bari-Active: a randomized controlled trial of a preoperative intervention to increase 
physical activity in bariatric surgery patients. Surg Obes Relat Dis. 2014 Jul 30;11(1):17780.

13. Bond DS, Thomas JG, King WC, Vithiananthan S, Trautvetter J, Unick JL, et al. Exercise improves quality of life in bariatric surgery candidates: Results from the BariActive trial. Obesity (Silver Spring). 2015 Jan 22.

14. Baillot A, Mampuya WM, Dionne IJ, Comeau E, Meziat-Burdin A, Langlois MF. Impacts of Supervised Exercise Training in Addition to Interdisciplinary Lifestyle Management in Subjects Awaiting Bariatric Surgery: a Randomized Controlled Study. Obes Surg. 2016 Apr 1; . [Epub ahead of print].

15. McCue M, Fairman A, Pramuka M. Enhancing quality of life through telerehabilitation. Phy Med Rehab Clin North Am. 2010 Feb;21(1):195-205.

16. Theodoros D, Russell T. Telerehabilitation: current perspectives. Stud Health Technol Inform. 2008;131:191-209.

17. Kairy D, Lehoux P, Vincent C, Visintin M. A systematic review of clinical outcomes, clinical process, healthcare utilization and costs associated with telerehabilitation. Disabil Rehabil. 2009;31(6):427-47. 
18. Tousignant M, Boissy $\mathrm{P}$, Corriveau H, Moffet H. In home telerehabilitation for older adults after discharge from an acute hospital or rehabilitation unit: A proof-of-concept study and costs estimation. Disabil Rehabil Assistive Technol. 2006 Sep;1(4):209-16.

19. Tousignant M, Boissy P, Moffet H, Corriveau H, Cabana F, Marquis F, et al. Patients' satisfaction of healthcare services and perception with in-home telerehabilitation and physiotherapists' satisfaction toward technology for post-knee arthroplasty: an embedded study in a randomized trial. Telemed J e-health. $2011 \mathrm{Jun} ; \mathbf{1 7}(5): 376-82$.

20. Tousignant M, Moffet H, Boissy P, Corriveau H, Cabana F, Marquis F. A randomized controlled trial of home telerehabilitation for post-knee arthroplasty. $J$ Telemed Telecare. 2011;17(4):195-8.

21. Mbuila Mampuya W. Cardiac rehabilitation past, present and future: an overview. Cardiovasc Diagn Ther. 2012;2:38-49.

22. Piotrowicz E, Piotrowicz R. Cardiac telerehabilitation: current situation and future challenges. Eur J Prevent Cardiol. 2013 Jun;20(2 Suppl):12-6.

23. Boissy P, Tousignant M, Moffet H, Nadeau S, Brière S, Mérette C, et al. Conditions of use, reliability and quality of audio/video-mediated communications during in-home rehabilitation teletreament for post-knee arthroplasty. J Telemed e-Health 2016; . [Epub ahead of print]. 
24. Moffet H, Tousignant M, Nadeau S, Merette C, Boissy P, Corriveau H, et al. InHome Telerehabilitation Compared with Face-to-Face Rehabilitation After Total Knee Arthroplasty: A Noninferiority Randomized Controlled Trial. J Bone Joint Surg Am. 2015 Jul 15;97(14):1129-41.

25. Tousignant M, Moffet H, Nadeau S, Merette C, Boissy P, Corriveau H, et al. Cost analysis of in-home telerehabilitation for post-knee arthroplasty. J Med Internet Res 2015;17(3):e83.

26. Wu G, Keyes LM. Group tele-exercise for improving balance in elders. Telemed J e-health. 2006 Oct;12(5):561-70.

27. Holland AE, Hill CJ, Rochford P, Fiore J, Berlowitz DJ, McDonald CF. Telerehabilitation for people with chronic obstructive pulmonary disease: feasibility of a simple, real time model of supervised exercise training. J Telemed Telecare. 2013 Jun;19(4):222-6.

28. Marquis N, Larivee P, Saey D, Dubois MF, Tousignant M. In-Home Pulmonary Telerehabilitation for Patients with Chronic Obstructive Pulmonary Disease: A Preexperimental Study on Effectiveness, Satisfaction, and Adherence. Telemed J e-health. 2015 Nov;21(11):870-9. 
29. Tousignant M, Corriveau H, Kairy D, Berg K, Dubois MF, Gosselin S, et al. Tai Chi-based exercise program provided via telerehabilitation compared to home visits in a post-stroke population who have returned home without intensive rehabilitation: study protocol for a randomized, non-inferiority clinical trial. Trials. 2014;15:42.

30. Armstrong R, Bull F. Development of the World Health Organization Global Physical Activity Questionnaire. J Public Health. 2006;14:66-70.

31. Finkelstein SM, Speedie SM, Demiris G, Veen M, Lundgren JM, Potthoff S. Telehomecare: quality, perception, satisfaction. Telemed J e-health. 2004 Summer;10(2):122-8.

32. Demiris G, Speedie SM, Finkelstein S. Change of patients' perceptions of TeleHomeCare. Telemed J e-health. 2001 Fall;7(3):241-8.

33. Yip MP, Chang AM, Chan J, MacKenzie AE. Development of the Telemedicine Satisfaction Questionnaire to evaluate patient satisfaction with telemedicine: a preliminary study. J Telemed Telecare. 2003;9(1):46-50.

34. Gagnon M, Hebert R, Dube M, Dubois MF. Development and validation of the Health Care Satisfaction Questionnaire (HCSQ) in elders. J Nurs Meas. 2006 Winter;14(3):190-204. 
35. Gibbons RJ, Balady GJ, Bricker JT, Chaitman BR, Fletcher GF, Froelicher VF, et al. ACC/AHA 2002 guideline update for exercise testing: summary article. A report of the American College of Cardiology/American Heart Association Task Force on Practice Guidelines (Committee to Update the 1997 Exercise Testing Guidelines). J Am Coll Cardiol. 2002 Oct 16;40(8):1531-40.

36. ATS statement: guidelines for the six-minute walk test. Am J Respir Crit Care Med. 2002 Jul 1;166(1):111-7.

37. Therrien F, Marceau P, Turgeon N, Biron S, Richard D, Lacasse Y. The laval questionnaire: a new instrument to measure quality of life in morbid obesity. Health Qual Life Outcomes.9:66.

38. Larsen JK, Geenen R, van Ramshorst B, Brand N, Hox JJ, Stroebe W, et al. Binge eating and exercise behavior after surgery for severe obesity: a structural equation model. Int J Eat Disord. 2006 Jul;39(5):369-75.

39. Piron L, Turolla A, Tonin P, Piccione F, Lain L, Dam M. Satisfaction with care in post-stroke patients undergoing a telerehabilitation programme at home. J Telemed Telecare. 2008;14(5):257-60. 


\section{Tables with legends}

Table 1. Baseline characteristics of participants

\begin{tabular}{llll}
\hline & TelePreSET $(\mathbf{n}=\mathbf{6})$ & PreSET $(\mathbf{n = 1 2})$ & Usual care $(\mathbf{n}=11)$ \\
\hline Age (years) & $44.8(39.6-54.7)$ & $45.1(38.6-55.1)$ & $43.5(37.0-46.2)$ \\
Body mass index $\left(\mathrm{kg} / \mathrm{m}^{2}\right)$ & $46.6(39.2-48.5)$ & $44.4(40.7-53.5)$ & $48.4(40.6-53.3)$ \\
Neck circumference $(\mathrm{cm})$ & $40.6(38.5-44.1)$ & $39.1(37.6-44.2)$ & $41.0(39.0-45.0)$ \\
Fat Mass (\%) & $49.7(48.8-52.0)$ & $50.4(47.4-53.3)$ & $50.6(46.6-52.0)$ \\
Systolic blood pressure (mmHg) & $121.5(112.4-134.3)$ & $121.3(111.9-133.8)$ & $119.5(112.0-124.5)$ \\
Diastolic blood pressure (mmHg) & $76.5(71.4-89.5)$ & $73.0(66.1-81.0)$ & $75.0(70.5-78.0)$ \\
Resting heart rate (beat/min) & $80.5(75.0-83.8)$ & $72.0(65.5-82.0)$ & $78.0(67.0-81.0)$ \\
Hypertension (\%) & 50.0 & 58.3 & 18.2 \\
Dyslipidemia (\%) & 16.7 & 50.0 & 45.5 \\
Type 2 diabetes (\%) & 33.3 & 33.3 & 36.4 \\
Prediabetes (\%) & 0.0 & 25.0 & 9.1 \\
Sleep apnea (\%) & 66.7 & 58.3 & 54.5 \\
Heart and vascular diseases (\%) & 16.7 & 16.7 & 9.1 \\
Asthma (\%) & 16.7 & 25.0 & 9.1 \\
Hypothyroidism (\%) & 33.3 & 0.0 & 36.4 \\
Anxiety and/or depression (\%) & 50.0 & 33.3 & 27.3 \\
Arthritis and/or arthrosis (\%) & 33.3 & 33.3 & 81.3 \\
Active (\%)* & 83.3 & 66.7 & $1413(929-3996)$ \\
Total PA energy expenditure & $1015(543-1129)$ & $900(419-1814)$ & \\
(METmin/week) & & & \\
\hline
\end{tabular}

*= following PA recommendation (600 METmin/week); PreSET=pre-surgery exercise training; TelePreSET=PreSET in telehealth

No significant difference between groups.

Data are presented with median $\left(25^{\text {th }}-75^{\text {th }}\right.$ percentiles $)$. 
Table 2. Baseline and 12-weeks change of physical fitness among participants.

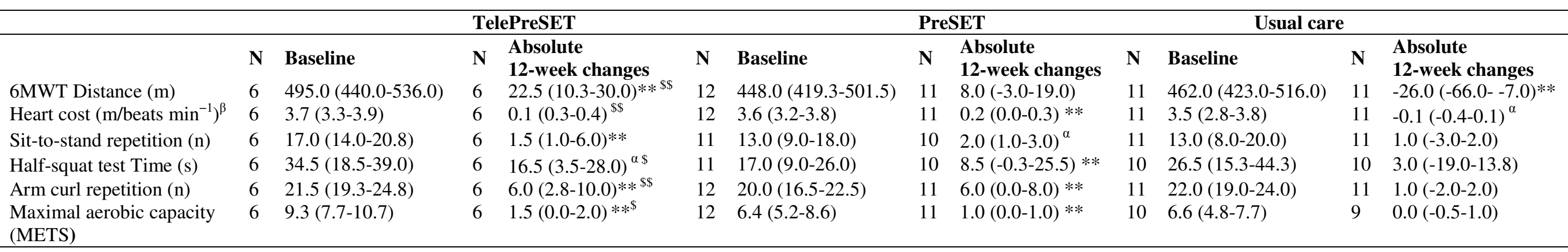

$* *=\mathrm{p}<0.05$ compared to baseline; ${ }^{\alpha}=\mathrm{p} \leq 0.09$ compared to baseline

$\$ \$=\mathrm{p}<0.05$ compared to usual care group; ${ }^{\$}=\mathrm{p} \leq 0.09$ compared to usual care group

$\beta=$ calculated using the $6 \mathrm{MWT}$ distance divided by $6 \mathrm{MWT}$ mean heart rate

6MWT = 6-minute walk test; PreSET=pre-surgery exercise training; TelePreSET=PreSET in telehealth

No significant baseline difference between groups. No significant 12-week change difference between TelePreSET and PreSET groups. Data are presented with median $\left(25^{\text {th }}-75^{\text {th }}\right.$ percentiles).

Missing data: Half-squat and sit-to-stand tests' missing data are explained by the inability to perform the test by participants because of knee pain.

One PreSET participant did not come to the final assessment because of pneumonia. One usual care participant did not perform any symptomlimited cardiac exercise because of functional limitations due to knee prosthesis. Another usual care participant refused to perform the final symptom-limited cardiac exercise due to major decline in her functional capacities (6MWT was already very difficult). 